\title{
High stability of properties in morphotropic phase boundary $\mathrm{Bi}_{0.5} \mathrm{Na}_{0.5} \mathrm{TiO}_{3}-\mathrm{BaTiO}_{3}$ piezoceramics
}

\author{
Rodrigo Machado, ${ }^{1,2}$ Diego A. Ochoa, ${ }^{1}$ Valdeci B. dos Santos, ${ }^{1,3}$ Elena Cerdeiras, ${ }^{4}$ \\ Lourdes Mestres ${ }^{4}$, and José E. García, ${ }^{1, *}$ \\ ${ }^{1}$ Departament de Física, Universitat Politècnica de Catalunya - BarcelonaTech, 08034 Barcelona, Spain. \\ ${ }^{2}$ Instituto de Física Rosario, Universidad Nacional de Rosario, 2000 Rosario, Argentina. \\ ${ }^{3}$ Coordenação de Engenharia de Materiais, Universidade Federal do Piauí, 64049-550 Teresina, Brazil. \\ ${ }^{4}$ Departament de Química Inorgànica i Orgànica, Secció de Química Inorgànica, Universitat de Barcelona, \\ 08028 Barcelona, Spain.
}

\section{Abstract}

The $(1-x) \mathrm{Bi}_{0.5} \mathrm{Na}_{0.5} \mathrm{TiO}_{3}-x \mathrm{BaTiO}_{3} \quad(\mathrm{BNT}-x \mathrm{BT})$ system with composition at its morphotropic phase boundary (MPB) has received significant attention because of their attractive properties as lead-free piezoceramics. Although the basic properties of this system are well-established, reports about the stability of the functional properties of these piezoelectric materials are still lacking. A study on the dielectric and piezoelectric properties of BNT- $x \mathrm{BT}$ close to their MPB, with emphasis on material response under high electric field or mechanical stress, is performed in this work. The results indicate that the BNT-BT system exhibits a high stability of dielectric and piezoelectric properties, making it potentially interesting for specific applications. A direct correlation between piezoelectric properties and nonlinear response is evidenced for a wide number of piezoceramics, which is expected due to the extrinsic nature of the piezoelectric response. Finding compositions that show high electromechanical properties and low nonlinear behaviour is a challenge in the search for competitive lead-free piezoceramics.

Keywords: Ferroelectrics; Piezoelectrics materials; Piezoelectric properties; Nonlinear behavior; BNT-BT.

* Corresponding author.

E-mail address: jose.eduardo.garcia@upc.edu (J.E. García). 


\section{Introduction}

Lead-based perovskite-type piezoceramics with compositions close to the morphotropic phase boundary (MPB) have been extensively used as active materials for transducer applications owing to their exceptional functional properties. Despite the excellent properties exhibited by lead-based piezoceramics, and in particular lead zirconate titanate (PZT) based compositions, these materials present some drawbacks that the scientific community has been trying to solve. One critical disadvantage of lead-based piezoceramics is their high lead content, which creates hazards during materials processing and poses a problem of waste disposal. As a consequence, and due to recent regulatory laws about the use of lead, enormous efforts have been made in the last decade in the search for high performance lead-free piezoceramics [1]. A significant number of binary or ternary systems with MPB have been synthesized and characterized, among which high piezoelectric properties and coupling coefficients have been achieved in $\left(\mathrm{K}_{1 / 2} \mathrm{Na}_{1 / 2}\right) \mathrm{NbO}_{3}(\mathrm{KNN})$ based compositions [2]. However, KNN-based piezoceramics exhibit a markedly nonlinear response, i.e. instability of properties, which limits their applications [3]. Compositional engineering by doping has been the traditional method used to minimize nonlinear effects, but so far only with moderate success in lead-free compositions.

The (1- $x) \mathrm{Bi}_{0.5} \mathrm{Na}_{0.5} \mathrm{TiO}_{3}-x \mathrm{BaTiO}_{3}(\mathrm{BNT}-x \mathrm{BT})$ system with composition around MPB $(0.05 \leq x \leq 0.08)$ has received significant attention because of its attractive properties $\left(d_{33}\right.$ $>150 \mathrm{pC} / \mathrm{N}, k_{\mathrm{p}}>20 \%, \tan \delta<2 \%, E_{\mathrm{c}}<30 \mathrm{kV} / \mathrm{mm}$ ) [4-6]. Although the basic properties of this system are well-established, and different synthesis routes [6-9] and doping strategies [10-16] have been explored in order to improve the properties, reports about the stability of the functional properties of these piezoelectric materials are still lacking. In this perspective, this work reports the results of a study on dielectric and piezoelectric 
properties of undoped BNT- $x \mathrm{BT}$ close to MPB ( $x=0.06$ and 0.07$)$, with emphasis on material response under high, but sub-switching, electric field or mechanical stress (i.e. nonlinear response), which reflect the real working conditions of these materials in devices. Some aspects related to the piezoelectric response are also discussed in terms of the Rayleigh model.

\section{Experimental}

$(1-x) \mathrm{Bi} 0.5 \mathrm{Na}_{0.5} \mathrm{TiO}_{3}-x \mathrm{BaTiO}_{3}(\mathrm{BNT}-x \mathrm{BT})$ ceramics with $\mathrm{x}=0.06$ and 0.07 were prepared by a solid-state reaction route. Details about the materials processing and some basic properties of the synthesized materials can be found in "Supplementary Information". Nonlinear dielectric response measurements were carried out in a plane capacitor configuration by using a capacitance comparator bridge specially designed for this type of measurement, as described in detail elsewhere [17]. Permittivity was measured by applying a $1 \mathrm{kHz}$ driving ac electric field from 0.06 to $0.6 \mathrm{kV} / \mathrm{mm}$, thereby ensuring a sub-switching range. Nonlinear longitudinal piezoelectric responses (dynamic piezoelectric coefficient) were measured by a Berlincourt-type method, similar to those previously described in the literature [18].

\section{Results and discussion}

Fig. 1 shows the electric field amplitude dependence of the real and imaginary relative dielectric permittivity at $1 \mathrm{kHz}$ and at room temperature for the materials under study. The low-field values of permittivity are reported in "Supplementary Information". As may be observed, both the real and imaginary parts of the permittivity increase with the increase in the electric field amplitude, and show an expected nonlinear dielectric response [19]. Nevertheless, although a certain instability in the dielectric response is 
exhibited in BNT- $x \mathrm{BT}$, the relative increase in the permittivity of this system is significantly low. Taking as a reference the increase in $\varepsilon^{\prime}$ at $E_{0}=0.30 \mathrm{kV} / \mathrm{mm}$, this is lower than $2 \%$ for BNT- $x$ BT, while in a well-known lead-free, KNN-based composition it is $35 \%$ [3]. In fact, the increment of $\varepsilon^{\prime}$ at $E_{0}=0.30 \mathrm{kV} / \mathrm{mm}$ is $50 \%$ for a soft commercial PZT (Pz27, from Ferroperm A/S) and $6 \%$ for a hard commercial PZT (PZT4, from Morgan Matroc Ltd.) [17]. It is important to point out that hard PZT are compositional engineered PZT-based materials developed to achieve a high stability of their properties, among other attributes. Thus, the results indicate that the BNT-BT system exhibits a high stability in the dielectric response, which is a not common feature in lead-free piezoceramics.

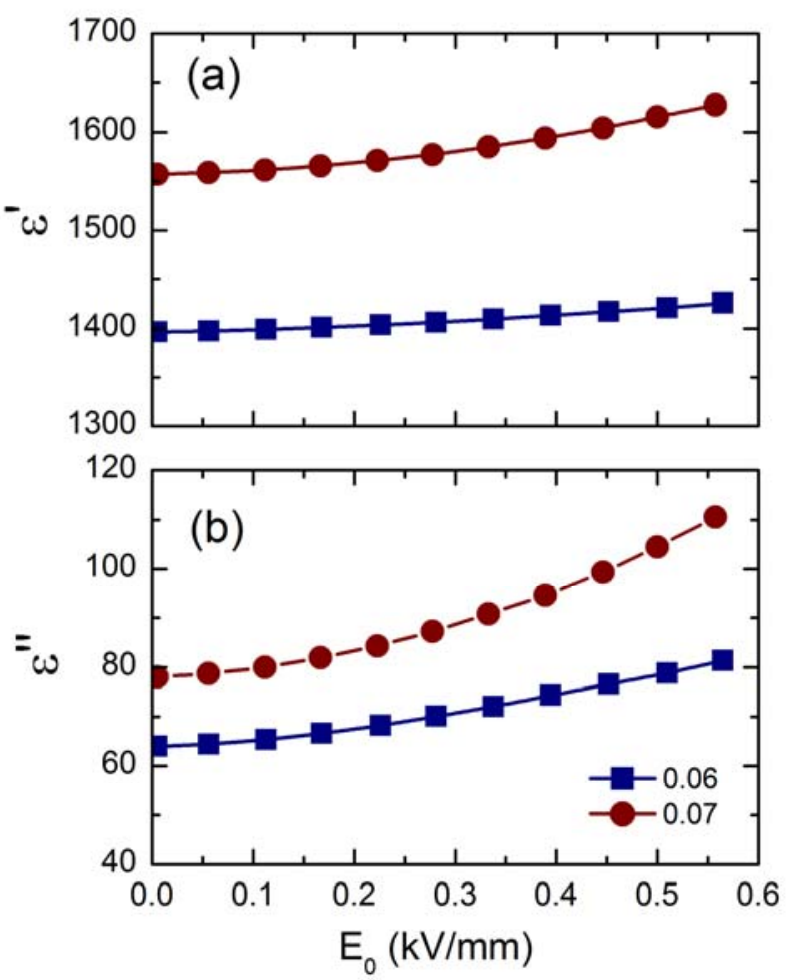

Fig. 1. Nonlinear dielectric response of $(1-x) \mathrm{Bi}_{0.5} \mathrm{Na}_{0.5} \mathrm{TiO}_{3}-x \mathrm{BaTiO}_{3}$, for $x=0.06$ and 0.07 , at 1 $\mathrm{kHz}$ and at room temperature. The real and the imaginary parts of the relative permittivity as a function of the applied ac electric field amplitude are shown in (a) and (b), respectively.

The observed stability of dielectric properties in the BNT- $x$ BT system suggests that a high stability of piezoelectric properties may be achieved in these materials, since a 
common origin of the nonlinear response, either dielectric or piezoelectric, is expected in perovskite piezoceramics [20,21]. From this perspective, Fig. 2a shows the dependence of the longitudinal piezoelectric coefficient $d_{33}$ with the amplitude $T_{0}$ of the applied dynamical stress for different 'bias' uniaxial compressive stress $T_{\mathrm{DC}}$. The figure shows this dependence for $x=0.06$, which is similar to the behaviour for $x=0.07$. Two main features of piezoelectric response can be highlighted. First, one may observe that when the amplitude $T_{0}$ increases $d_{33}$ also increases, thereby displaying a nonlinear behaviour for any $T_{\mathrm{DC}}$. Second, $d_{33}$ decreases for any amplitude $T_{0}$ when $T_{\mathrm{DC}}$ is increased, which may be explained as a result of the domain wall clamping effect [22]. In particular, the higher the $T_{\mathrm{DC}}$, the lower the $d_{33}$ at zero-stress amplitude $\left(d_{33 \mathrm{~L}}\right)$, as shown in Fig. $2 \mathrm{~b}$.
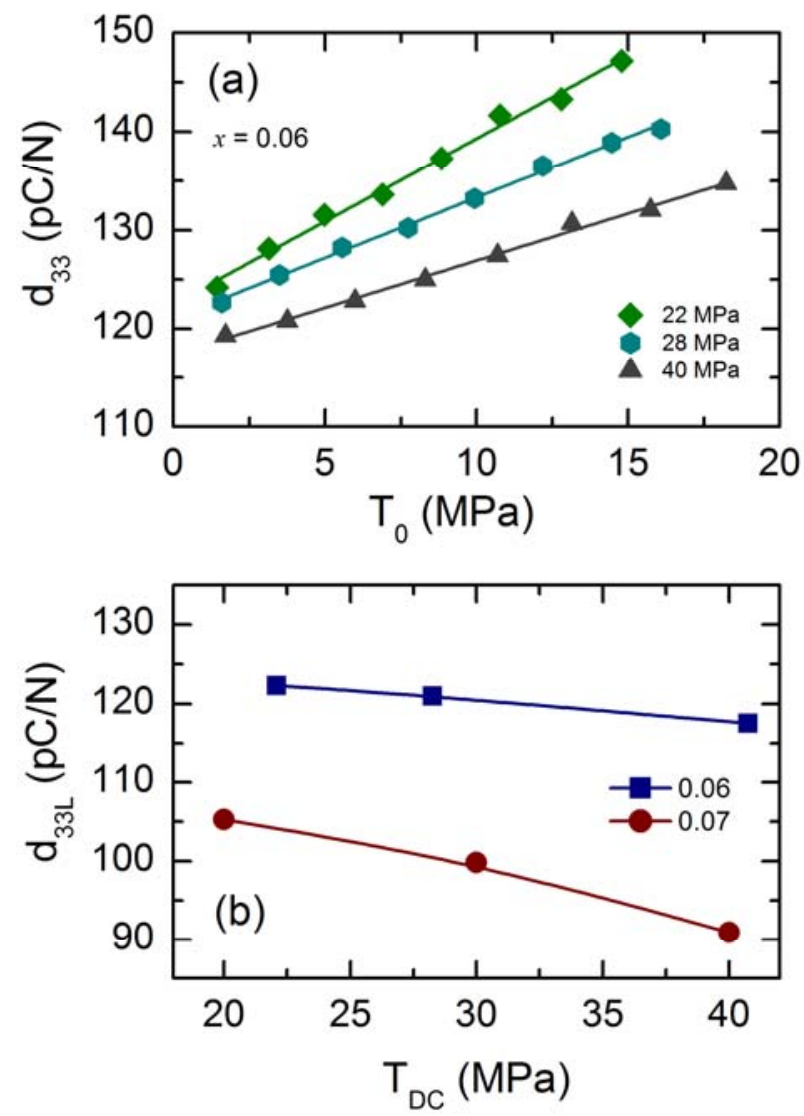

Fig. 2. Direct piezoelectric coefficient of $(1-x) \mathrm{Bi}_{0.5} \mathrm{Na}_{0.5} \mathrm{TiO}_{3}-x \mathrm{BaTiO}_{3}$, at $1 \mathrm{~Hz}$ and at room temperature, as a function of: (a) the amplitude of the applied dynamical stress for $x=0.06$; (b) the uniaxial compression pre-stress for $x=0.06$ and 0.07 . In (b) $d_{33 \mathrm{~L}}$ represents the direct piezoelectric coefficient at zero-stress amplitude $\left(T_{0}=0\right)$. 
As may be observed in Fig. 2a, the piezoelectric coefficient $d_{33}$ increases linearly as dynamic stress amplitude $T_{0}$ increases for all $T_{\mathrm{DC}}$, which is in agreement with the Rayleigh law. This model has been satisfactorily used to describe the nonlinear piezoelectric response in ferroelectric materials $[21,23,24]$. According to this model, the dynamic stress amplitude dependence of the direct longitudinal piezoelectric coefficient can be expressed as:

$$
d_{33}=d_{33 L}+\alpha_{d} T_{0}
$$

where $\alpha_{d}$ is called the Rayleigh coefficient. This is a useful parameter to quantify the instability of the piezoelectric response. The $\alpha d$ value is easily obtained by a linear fitting of $d_{33}$ versus $T_{0}$ curve, for a given $T_{\mathrm{DC}}$.

It is well known that nonlinear response is related to the extrinsic contribution and that this is the main contribution to the material response in perovskite piezoceramics at room temperature [19]. Thus, a correlation between piezoelectric properties and nonlinear response is expected in perovskite polycrystals; e.g., the larger the piezoelectric coefficient the larger the Rayleigh coefficient. Fig. 3 shows the values of the longitudinal piezoelectric coefficient correlated with the Rayleigh coefficient for some piezoceramics. The data are extracted from $d_{33}$ versus $T_{0}$ curves measured under a 'bias' uniaxial compressive stress of $20 \mathrm{MPa}$, at $1 \mathrm{~Hz}$ and at room temperature. As may be observed, compositions with a high piezoelectric coefficient exhibit higher values of Rayleigh coefficient, as is the case of the Pz27. A wide number of PZT-based materials are shown in Fig. 3 because the PZT system can be compositionally engineered in order to notably modify its properties. It is therefore possible to achieve such different materials as those denoted by PZT-Fe and PLZT 9/65/35. However, finding a composition whose properties are located in the region indicated as "desired properties" in Fig. 3 poses a challenge, since the nonlinear response is extrinsic in nature in these materials. It appears that lead- 
free piezoceramics are not unconnected with this issue, which is in line with the trend set by the lead-based compositions.

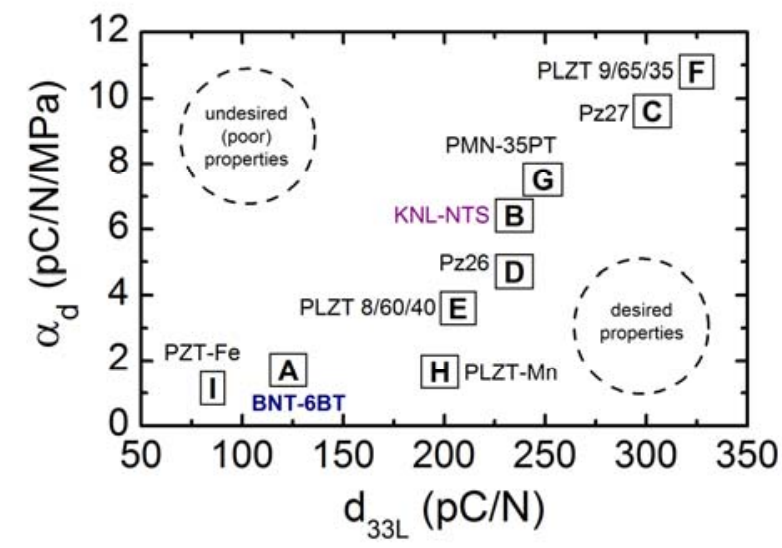

Fig. 3. Correlation between the Rayleigh coefficient and the zero-stress amplitude piezoelectric coefficient for some piezoelectric materials, measured at $1 \mathrm{~Hz}$ and under a uniaxial mechanical stress of $20 \mathrm{MPa}$. Data for A are extracted from Fig. 2(a); data for B, C and D are extracted from Fig. 1S (Supplementary Information); data for E, F, G, H and I are extracted from Refs. [2528].

\section{Conclusion}

Not only are high values of electromechanical coefficients desirable in the race to obtain competitive lead-free piezoelectric ceramics, but also a high stability of these properties is needed to effectively replace lead-based compositions in high power applications. In this context, BNT- $x \mathrm{BT}$ ceramics with a composition around the morphotropic phase boundary are studied in order to evaluate the performance of these materials when they work under a high electric field or mechanical stress. Results indicate that BNT- $x \mathrm{BT}$ exhibits a high stability of dielectric and piezoelectric responses, which may make this system suitable for specific applications. Since the origin of the piezoelectric response is extrinsic in nature, a direct correlation between the longitudinal piezoelectric coefficient and the Rayleigh coefficient is evidenced for a wide number of piezoceramics. Desirable properties of piezoceramics include a high and stable piezoelectric response together with low losses, which constitutes an ongoing challenge in material science. 


\section{Acknowledgements}

This work is supported by the MINECO (Spanish Government) project MAT201348009-C4-P-2. Dr. R.Machado and Dr. V.B dos Santos thank CONICET and CNPq, respectively, for their postdoctoral scholarships.

\section{References}

[1] J. Rödel, K.G. Webber, R. Dittmer, W. Jo, M. Kimura, D. Damjanovic, Transferring lead-free piezoelectric ceramics into application, J. Eur. Ceram. Soc. 35 (2015) $1659-1681$.

[2] J.-F. Li, K. Wang, F.-Y. Zhu, L.-Q. Cheng, F.-Z. Yao, (K,Na)NbO3-based lead-free piezoceramics: fundamental aspects, processing technologies, and remaining challenges, J. Am. Ceram. Soc. 96 (2013) 3677-3696.

[3] D.A. Ochoa, J.E. García, R. Pérez, V. Gomis, A. Albareda, F. Rubio-Marcos, J.F. Fernandez, Extrinsic contribution and non-linear response in lead-free KNNmodified piezoceramics, J. Phys. D. Appl. Phys. 42 (2009) 025402.

[4] C. Xu, D. Lin, K.W. Kwok, Structure, electrical properties and depolarization temperature of ( $\mathrm{Bi} 0.5 \mathrm{Na} 0.5) \mathrm{TiO}_{3}-\mathrm{BaTiO}_{3}$ lead-free piezoelectric ceramics, Solid State Sci. 10 (2008) 934-940.

[5] B. Parija, T. Badapanda, S. Panigrahi, T.P. Sinha, Ferroelectric and piezoelectric properties of $(1-\mathrm{x})\left(\mathrm{Bi}_{0.5} \mathrm{Na}_{0.5}\right) \mathrm{TiO}_{3}-\mathrm{xBaTiO}_{3}$ ceramics, J. Mater. Sci. Mater. Electron. 24 (2012) 402-410.

[6] M. Chen, Q. Xu, B.H. Kim, B.K. Ahn, J.H. Ko, W.J. Kang, O.J. Nam, Structure and electrical properties of $\left(\mathrm{Na}_{0.5} \mathrm{Bi}_{0.5}\right)_{1-\mathrm{x}} \mathrm{Ba}_{\mathrm{x}} \mathrm{TiO}_{3}$ piezoelectric ceramics, J. Eur. Ceram. Soc. 28 (2008) 843-849.

[7] H. Yilmaz, G.L. Messing, S. Trolier-McKinstry, (Reactive) Templated grain growth of textured sodium bismuth titanate $\left(\mathrm{Na}_{1 / 2} \mathrm{Bi}_{1 / 2} \mathrm{TiO}_{3}-\mathrm{BaTiO}_{3}\right)$ ceramics-I Processing, J. Electroceram. 11 (2003) 207-215.

[8] D. Zhou, H. Li, S. Gong, Y. Hu, K. Han, Sodium bismuth titanate-based lead-free piezoceramics prepared by aqueous gelcasting, J. Am. Ceram. Soc. 91 (2008) 2792-2796. 
[9] E. Mercadelli, A. Sanson, C. Capiani, A.L. Costa, C. Galassi, Influence of the synthesis route on the properties of BNBT ceramics, Process. Appl. Ceram. 3 (2009) 73-78.

[10] H.-D. Li, C.-D. Feng, P.-H. Xiang, Electrical properties of $\mathrm{La}^{3+}$-doped (Na0.5Bi 0.5)0.94Ba0.06TiO3 ceramics, Jpn. J. Appl. Phys. 42 (2003) 7387-7391.

[11] X.X. Wang, H.L.W. Chan, C.L. Choy, (Bi0.5Na0.5)0.94Ba0.06TiO3 lead-free ceramics with simultaneous addition of $\mathrm{CeO}_{2}$ and $\mathrm{La}_{2} \mathrm{O}_{3}$, Appl. Phys. A. 80 (2003) 333-336.

[12] X.Y. Zhou, H.S. Gu, Y. Wang, W.Y. Li, T.S. Zhou, Piezoelectric properties of Mndoped ( $\left.\mathrm{Na}_{0.5} \mathrm{Bi}_{0.5}\right)_{0.92} \mathrm{Ba}_{0.08} \mathrm{TiO}_{3}$ ceramics, Mater. Lett. 59 (2005) 1649-1652.

[13] C. Zhou, X. Liu, W. Li, C. Yuan, Dielectric and piezoelectric properties of $\mathrm{Y}_{2} \mathrm{O}_{3}$ doped (Bi0.5Na0.5)0.94Ba0.06 $\mathrm{TiO}_{3}$ lead-free piezoelectric ceramics, Mater. Res. Bull. 44 (2009) 724-727.

[14] P. Fu, Z. Xu, R. Chu, W. Li, G. Zang, J. Hao, Piezoelectric, ferroelectric and dielectric properties of $\mathrm{La}_{2} \mathrm{O}_{3}$-doped $\left(\mathrm{Bi}_{0.5} \mathrm{Na}_{0.5}\right)_{0.94} \mathrm{Ba}_{0.06} \mathrm{TiO}_{3}$ lead-free ceramics, Mater. Des. 31 (2010) 796-801.

[15] Q. Li, H. Wang, H. Fan, C. Long, X. Liu, Ferroelectric, electromechanical, and dielectric properties of $\left(\mathrm{Na} 0.5 \mathrm{Bi}_{0.5}\right)_{0.94} \mathrm{Ba}_{0.06} \mathrm{TiO}_{3}$ co-doped $\mathrm{MnO}_{2}$ and $\mathrm{La}_{2} \mathrm{O}_{3}$ leadfree ceramics, J. Mater. Sci. 49 (2013) 211-217.

[16] S. Prasertpalichat, D.P. Cann, Hardening in non-stoichiometric (1-x)Bi0.5Na0.5 $\mathrm{TiO}_{3}-\mathrm{xBaTiO} 3$ lead-free piezoelectric ceramics, J. Mater. Sci. 51 (2016) 476-486.

[17] J.E. García, R. Pérez, A. Albareda, High electric field measurement of dielectric constant and losses of ferroelectric ceramics, J. Phys. D. Appl. Phys. 34 (2001) $3279-3284$.

[18] A. Barzegar, D. Damjanovic, N. Setter, The effect of boundary conditions and sample aspect ratio on apparent $d_{33}$ piezoelectric coefficient determined by direct quasistatic method, IEEE Trans. Ultrason. Ferroelectr. Freq. Control. 51 (2004) $262-70$.

[19] J.E. García, Extrinsic contribution and instability properties in lead-based and leadfree piezoceramics, Materials 8 (2015) 7821-7836.

[20] S. Li, W. Cao, L.E. Cross, The extrinsic nature of nonlinear behavior observed in lead zirconate titanate ferroelectric ceramic, J. Appl. Phys. 69 (1991) 7219-7224.

[21] D. A. Hall, Nonlinearity in piezoelectric ceramics, J. Mater. Sci. 36 (2001) 45754601 . 
[22] M. Demartin, D. Damjanovic, Dependence of the direct piezoelectric effect in coarse and fine grain barium titanate ceramics on dynamic and static pressure, Appl. Phys. Lett. 68 (1996) 3046-3048.

[23] D. Damjanovic, M. Demartin, The Rayleigh law in piezoelectric ceramics, J. Phys. D. Appl. Phys. 29 (1996) 2057-2060.

[24] D. Damjanovic, Stress and frequency dependency of the direct piezoelectric effect in ferroelectric ceramics, J. Appl. Phys. 82 (1997) 1788-1797.

[25] J.D.S. Guerra, J.E. García, D.A. Ochoa, A. Pelaíz-Barranco, O. García-Zaldívar, F. Calderón-Piñar, Interrelationship between phase transition characteristics and piezoelectric response in lead lanthanum zirconate titanate relaxor ceramics, J. Mater. Sci. 47 (2012) 5715-5720.

[26] E. Perez-Delfin, J.E. García, D.A. Ochoa, R. Pérez, F. Guerrero, J.A. Eiras, Effect of Mn-acceptor dopant on dielectric and piezoelectric responses of lead lanthanum zirconate titanate piezoceramics, J. Appl. Phys. 110 (2011) 034106.

[27] J.E. Garcia, J.D.S. Guerra, E.B. Araújo, R. Perez, Domain wall contribution to dielectric and piezoelectric responses in $0.65 \mathrm{~Pb}\left(\mathrm{Mg}_{1 / 3} \mathrm{Nb}_{2 / 3}\right)-0.35 \mathrm{PbTiO}_{3}$ ferroelectric ceramics, J. Phys. D. Appl. Phys. 42 (2009) 115421.

[28] D.A. Ochoa, J.E. García, I. Tamayo, V. Gomis, D. Damjanovic, R. Perez, Effect of uniaxial compressive stress on dielectric and piezoelectric responses in lead zirconate titanate based ceramics, J. Am. Ceram. Soc. 95 (2012) 1656-1660. 


\section{High stability of properties in morphotropic phase boundary $\mathrm{Bi}_{0.5} \mathrm{Na}_{0.5} \mathrm{TiO}_{3}-\mathrm{BaTiO}_{3}$ piezoceramics \\ R. Machado, D. A. Ochoa, V. B. dos Santos, E. Cerdeiras, L. Mestres, and J. E. García}

\section{Supplementary information}

\section{Details of the synthesis and basic properties of the materials}

$(1-x) \mathrm{Bi}_{0.5} \mathrm{Na}_{0.5} \mathrm{TiO}_{3}-x \mathrm{BaTiO}_{3}(\mathrm{BNT}-x \mathrm{BT})$ ceramics with $\mathrm{x}=0.06$ and 0.07 were prepared by a solid-state reaction route. $\mathrm{Bi}_{2} \mathrm{O}_{3}(99.9 \%), \mathrm{Na}_{2} \mathrm{CO}_{3}(99.5 \%), \mathrm{BaCO}_{3}(99 \%)$, and $\mathrm{TiO}_{2}$ (99.9\%) were used as the starting raw materials. The raw reagents were weighed according to the desired composition and ball-milled with yttria-stabilized zirconia balls in absolute ethanol medium at $300 \mathrm{rpm}$ for $8 \mathrm{~h}$. The solution was dispersed and then dried. The obtained powders were calcined at $700^{\circ} \mathrm{C}$ for $2 \mathrm{~h}$ and then milled again for $8 \mathrm{~h}$. The final powders were mixed with a binder solution, dried and then pressed at $700 \mathrm{MPa}$ to produce disc-shaped (13 $\mathrm{mm}$ diameter and $0.5 \mathrm{~mm}$ thick) and bar-shaped (16 $\mathrm{mm}$ long, $4 \mathrm{~mm}$ wide and $0.8 \mathrm{~mm}$ thick) samples. The pellets were sintered in air at $1200 \circ \mathrm{C}$ for $2 \mathrm{~h}$ in covered platinum crucibles. As-sintered samples showed relative densities over 95\% measured by Archimedes' method.

Room temperature X-ray diffraction measurement of powder of sintered samples were carried out to verify the structure of the studied compositions. The results show a pure perovskite structure with a mixture of rhombohedral and tetragonal crystal structures for both composition, as may be expected for BNT-BT system around their morphotropic phase boundary. Microstructural analysis by field-emission scanning electron microscopy revealed the polycrystalline nature of the samples with grains that are rounded, irregular shaped and inhomogeneous in size.

Gold electrodes were sputtered on parallel polished faces of the samples. A precision LCR meter (Agilent E4980A) was used to obtain the real and imaginary parts of the permittivity of unpoled samples at selected frequencies from $100 \mathrm{~Hz}$ to $1 \mathrm{MHz}$. Samples were then poled in a silicone bath at $25 \stackrel{\circ}{\circ} \mathrm{C}$ under a dc electric field of $4 \mathrm{kV} / \mathrm{mm}$ for 30 minutes. Before measurements, samples were aged for a week in order to prevent the 
influence of aging processes. The static longitudinal piezoelectric coefficient was measured with a $d_{33}$ meter (KCF Technologies PM3500). A precision impedance analyzer (Agilent 4294A) was employed to measure resonance and anti-resonance frequencies of the main vibration extensional mode of bar-shape samples as well as their capacitance value at the frequency where the motional admittance is null. The elastic compliance $s^{E}{ }_{11}$, the piezoelectric coefficient $d_{31}$, and the electromechanical coupling factor $k_{31}$ were determined on the basis of IEEE standards. Table S1 summarizes some basic room temperature functional properties of the synthesized materials.

Table S1. Room temperature values of the dielectric constant, $\varepsilon^{\prime}$, dielectric losses, $\tan \delta$, elastic compliance, $s^{\mathrm{E}}{ }_{11}$, piezoelectric coefficients, $d_{33}$ and $d_{31}$, and electromechanical coupling factor $k_{31}$ of $(1-x) \mathrm{Bi}_{0.5} \mathrm{Na}_{0.5} \mathrm{TiO}_{3}-x \mathrm{BaTiO}_{3}$, for $x=0.06$ and 0.07 .

\begin{tabular}{lllllll}
\hline$x$ & $\varepsilon^{\prime}$ & $\begin{array}{l}\tan \delta \\
(\%)\end{array}$ & $\begin{array}{l}d_{33} \\
(\mathrm{pC} / \mathrm{N})\end{array}$ & $\begin{array}{l}d_{31} \\
(\mathrm{pC} / \mathrm{N})\end{array}$ & $\begin{array}{l}s^{E_{11}} \\
\left(10^{-12} / \mathrm{Pa}\right)\end{array}$ & $k_{31}$ \\
\hline 0.06 & 1397 & 4.6 & 147 & 34 & 8.5 & 0.17 \\
0.07 & 1557 & 5.0 & 180 & 37 & 8.0 & 0.17 \\
\hline
\end{tabular}

\section{Nonlinear piezoelectric response of some piezoelectric ceramics}

Figure $\mathbf{S 1}$ shows the dependence of the longitudinal piezoelectric coefficient $d_{33}$ with the amplitude $T_{0}$ of the applied dynamical stress, measured under a 'bias' uniaxial compressive stress of $20 \mathrm{MPa}$, at $1 \mathrm{~Hz}$ and at room temperature. The piezoelectric coefficient $d_{33}$ increases linearly as dynamic stress amplitude $T_{0}$ increases; thus, $d_{33}=d_{33 L}+\alpha_{d} T_{0}$. The values of $\alpha_{d}$ are obtained by a linear fitting of $d_{33}$ versus $T_{0}$ curve for each material. In the figure, $\mathrm{Pz} 26$ and $\mathrm{Pz} 27$ are commercial hard and soft $\mathrm{Pb}(\mathrm{Zr}, \mathrm{Ti}) \mathrm{O}_{3}-$ based materials (Ferroperm Piezoceramics S/A) and KNL-NTS is the well-known KNNbased composition $\left(\mathrm{K}_{0.44} \mathrm{Na}_{0.52} \mathrm{Li}_{0.04}\right)\left(\mathrm{Nb}_{0.86} \mathrm{Ta}_{0.10} \mathrm{Sb}_{0.06}\right) \mathrm{O}_{3}$. 


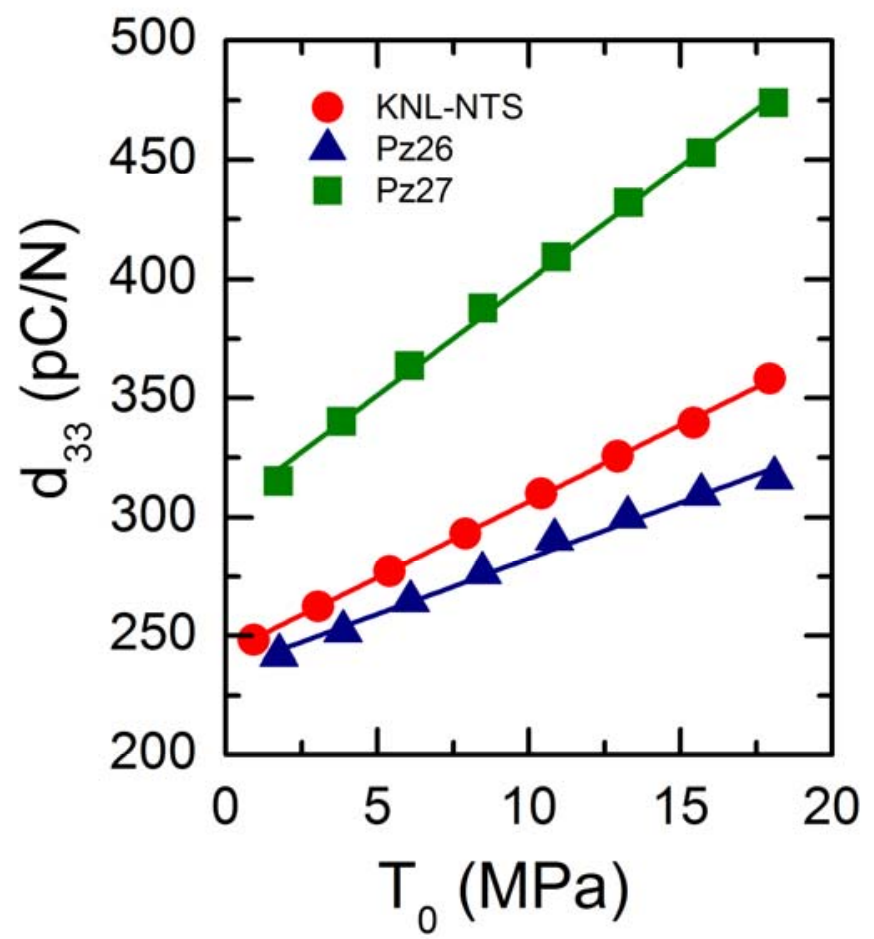

Fig. 2. Direct piezoelectric coefficient of some representative piezoceramics as a function of the amplitude of the applied dynamical stress, at $1 \mathrm{~Hz}$ and at room temperature. All measurement were performed under a uniaxial pre-stress of $20 \mathrm{Ma}$. 